\title{
Cooling Performance of Thermoelectric Cooling (TEC) and Applications: A review
}

\author{
'Aqilah Che Sulaiman', Nasrul Amri Mohd Amin"1, *, Mohd Hafif Basha', Mohd Shukry \\ Abdul Majid ${ }^{1}$, Nashrul Fazli bin Mohd Nasir ${ }^{1}$ and Izzuddin Zaman ${ }^{2}$ \\ ${ }^{1}$ School of Mechatronic Engineering, Universiti Malaysia Perlis, 02600 Arau, Perlis, Malaysia. \\ ${ }^{2}$ Faculty of Mechanical and Manufacturing Engineering, Universiti Tun Hussein Onn Malaysia, Parit \\ Raja, 86400 Batu Pahat, Johor, Malaysia.
}

\begin{abstract}
Thermoelectric cooling (TEC) is a new attractive method that is can be used as a temperature controller. Thermoelectric module (TEM) is a device that environmentally friendly utilizing for cooling and heating application such as heat pump and power generation. Therefore, the understanding of relation between electrical conductivity and heat conductivity of the TEC material is essentially to improve the coefficient of performance (COP) efficiency. The figure of merit is addressed by focusing the best material in TEC with different cooling material. The critical finding of TEC for this review paper is the higher the electrical conductivity and the lower thermal conductivity, the maximum the COP. Finally, the possiblity of the TEC application is reviewed according to the advantages of TEC such as high reliability, less maintenance and compact size that commercially found in large range of thermoelectric cooling system. $\mathrm{N}$
\end{abstract}

\section{Introduction}

Thermoelectric cooling (TEC) is a cooling system used in many application such as medical instrument, factory machine, electronic device and transportation tools. Thermoelectric module (TEM) is developed as an alternative eco-environmentally friendly application for heat pumps and power generator.

TEC is a reliable energy converter because it does not involve any moving particles, has no vibration or noise, need less maintenance and is portable [1]. The module is made up from a combination of thermal, electrical and semiconductor properties which is used to convert waste heat into electrical energy [2].

The electrical energy is then reused for cooling and heating application. The current that practically cross a junction of different electrical conductive textiles will cause a heating or cooling effect. The operation of TE module is based on three effect which is Peltier Effect, Seebeck Effect and Thomson Effect. The Peltier Effect is used in a cooler application whereas the Seebeck Effect is used as a power generator.

According to the cooling system experimented by Jean Charles Athanase Peltier in 1834, there are some idea to evaluate the cooling effectiveness performance of air conditioner that

*Corresponding author: nasrulamri.mohdamin@ unimap.edu.my 
integrated with TEC [3]. In this review paper, the importance of TEC is to reduce the electricity usage for cooling system.

\subsection{TEC WORKING PRINCIPLE}

The TEC module works by transferring the heat from hot side to cold side to the comparatively cold region area.

During the cooling or heating mode, the direct current passes from the n-type to p-type of semiconductor material where the cooling temperature interconnecting conductor decreased and heat is absorbed from the surroundings. The principal of the Peltier Effect involves the absorption of thermal energy from one dissimilar metal junction and release the thermal energy to another junction when the current flow into the circuit as shown in figure 1 .

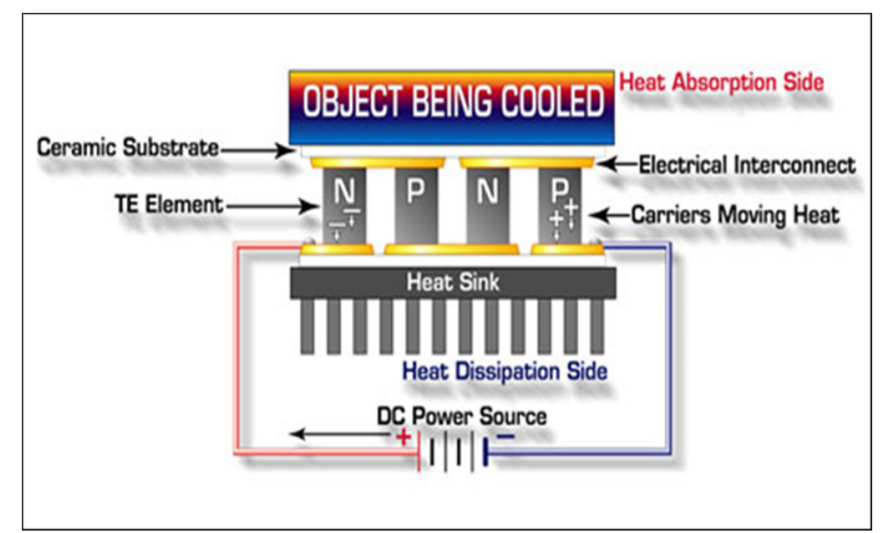

Fig. 1. Schematic diagram of cooling and heating of TEC [4].

The Principle of Seebeck Effect operates when two different metals are pairly joined and can maintain different temperatures. The generation of electromotive force (EMF) will exist when the current flow in the circuit $[1,2,5]$. Reversing the current flow may cause the reverses of hot and cold end.

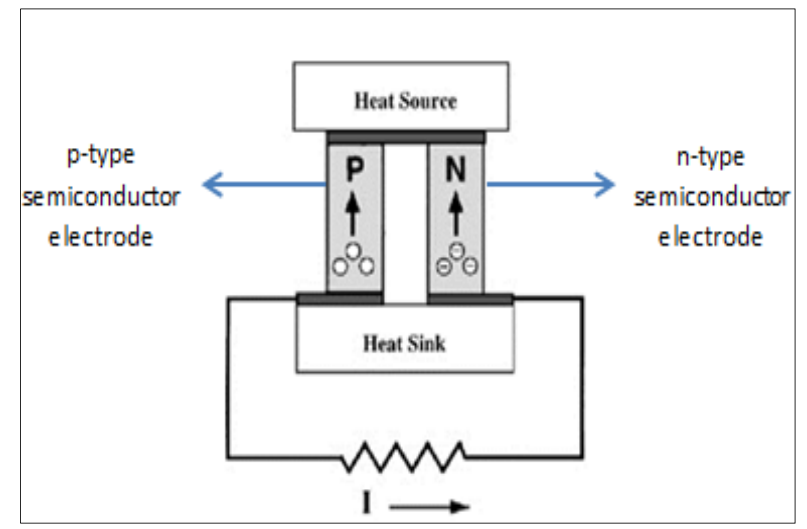

Fig. 2. Schematic diagram of TEC power generation [6].

As shown in figure 2, the electrodes were connected to each other and maintained at different temperatures so the EMF current flows across the circuit. This Seebeck Effect is 
directly proportional to the variance temperature when the current is generated through semiconductor material of TE module [7].

\section{Important Parameter of TEC}

The use of TEC is increasingly known because this device gives many advantages such as long lasting lifetime, huge scalability, ease of use, and less maintenance, especially in the manufacturing industry, transportation, military application, aerospace and medical instruments $[1,7]$.

The typical TE module is made up between two ceramic insulators that functions as electrical insulation for $\mathrm{p}$-type and n-type of thermoelement. The heat flow parallels between the ceramic and the p-type and n-type of thermoelement connected electrically in series as show in figure 3 .

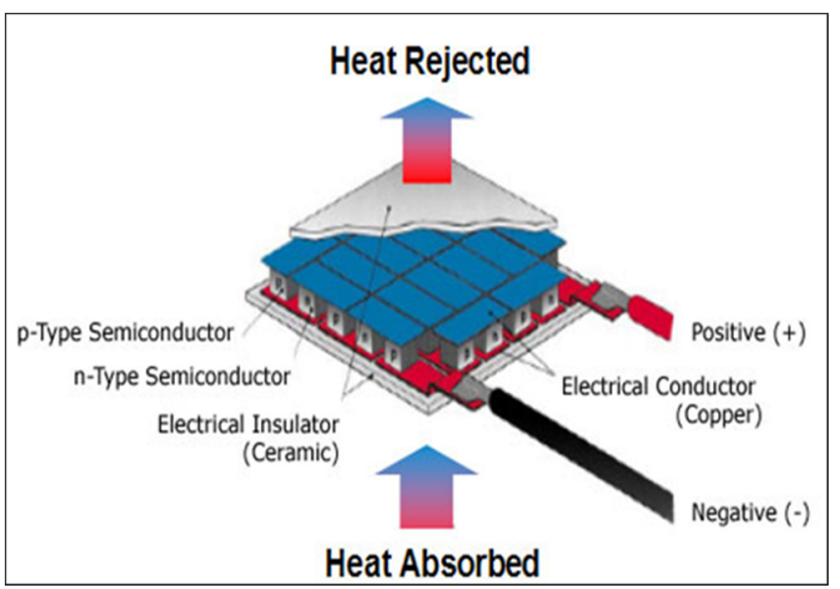

Fig. 3. A diagram of a TEC when electric current passed through a join of two ceramic insulators [8].

\subsection{TEC Material}

The quality of thermoelectric material is depending on thermoelectric power $\mathrm{S}$, the electrical conductivity $\sigma$ and the thermal conductivity $\kappa$. The electrical conductivity defines as the internal resistance and the related heating of the TEM which the thermal conductivity controlled the heat that flow through the module from decreasing the temperature different between hot side and cold side. However, the efficiency of the TEM depend on the thermoelectric figure of merit, ZT [9]:

$$
\mathrm{ZT}=\mathrm{S} 2 \sigma / \kappa
$$

The characterictic of thermoelectric efficiency is the ratio of electrical energy produced to the amount of thermal energy absorb from heat source [9]. The figure of merit ZT is the key of parameter that can increase the efficiency of TEC module. According to the TEC module, the element that have poor electrical conductivity is lead to produce low TEC effect [10]. In oder to increase the TEC efficiency, the figure of merit should be large. The larger figure of merit require low thermal conductivity but high electrical conductivity. Another parameter that relative to TEC efficiency is power factor which concentrate on material performance on electron while thermal conductivity produce at the end leg of TEC [10]. 


$$
\text { Power factor }=\sigma s^{\wedge} 2
$$

The conflict properties to develop the higher ZT and power factor of TEC material are optimized and considered as below:

- $\quad$ ZT about 1 is inefficient;

- $\quad \mathrm{ZT}=2$ is able to recover waste heat;

- $\mathrm{ZT}=4 / 5$ is able to match the refrigerator

Figure 4 show the ZT of Bismuth Telluride (BiTe) would able to reach the maximum value of $\mathrm{ZT} \sim 1$ because of the low temperature [11]. Nonetheless, the quasicrystalline alloys have always been used since its figure of merit is upper than $\mathrm{ZT}=1$ [9].

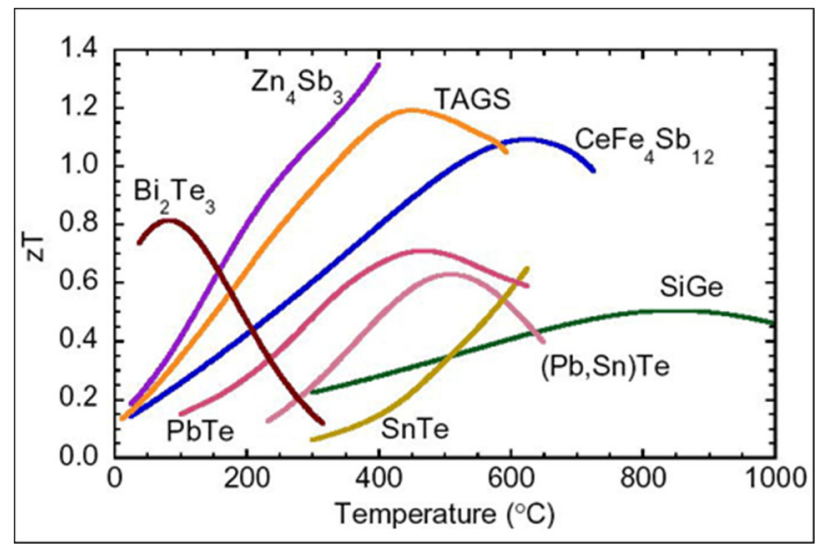

Fig. 4. The TE figure of merit ZT, depend on the electrical conductivity and the best figure of merit was found in Bismuth Telluride semiconductor [13].

The overall heat depletion and external thermal resistance are established along the thickness of module and the most favorable of the current operation. The researchers also state that to improve the thermal resistance by using thinner leg length and the higher figure of merit, the increase the reading of heat density.

\subsection{TEC Cooling Performance}

Various reseachers has obtain the cooling performance from TEC by optimizing the size, material, cooling capacity and the temperature different for improving the COP of TEC [10]. The cooling COP of TEC is depends on the current through the module and the temperature difference between two side of TEM. The COP of TEC is consider the temperature of cold side, $T \_c$ and temperature of hot side , $T \_h$ as equation below [13]:

$$
\operatorname{COP}_{C}=\frac{1}{\left(T_{h}-T_{C}\right)-1}=\frac{T_{C}}{T_{h}-T_{C}}
$$

\subsubsection{Low Cooling capacity}

Dongliang and Gang [11] conducted an experiment of thermoelectric cooling system with phase change material (PCM) heat storage. They found that the maximum COP value achieved is 1.22 at $7 \square$ temperature. The COP of TEC can highly perform when low cooling capacity and maximum capacity has achieved at $210 \mathrm{~W}$. The experimental result shows that 
the energy saving from this combination is $35.3 \%$ under an outdoor temperature range from 30-33 $\square$.

The heat sink will decrease the hot temperature. This process basically increases the performance efficiency of the system and the result state that the average COP of the cooling system is $56 \%$ and increase from 0.5 to 0.78 [12]. Both combination show that the TEC system has good integration with PCM for cooling application and at the same time increases the COP.

\subsubsection{Thermal Resistance}

The temperature that passes from hot side and cold side of TEC module usually can be used to evaluate the cooling performance.Huanget al [14] verified that the performance curve and basic properties of TEC were measured. Than the system selected the parameter design of thermoelectric cooler that used thermal resistance of the heat sink to remove out complicated heat transfer from the system. The optimum cooling capacity and optimum COP could be determined more accurately using the thermal resistance because it can release the waste heat from cold side to the ambient.

\subsubsection{Multistage TE Module}

The COP of the cooling system will decrease when the dissimilar temperature is increased, but the usage of multistage thermoelectric module can improve its performance [2,15-16].

The researchers reported that the difference performance between single module and multistage module is about $35.8 \%$. The multistage module may lead to the maximization of the cooling efficiency with appropriate number of stages with cascaded thermoelectric cooler modules. Figure 5 is the picture of single module of TEC.

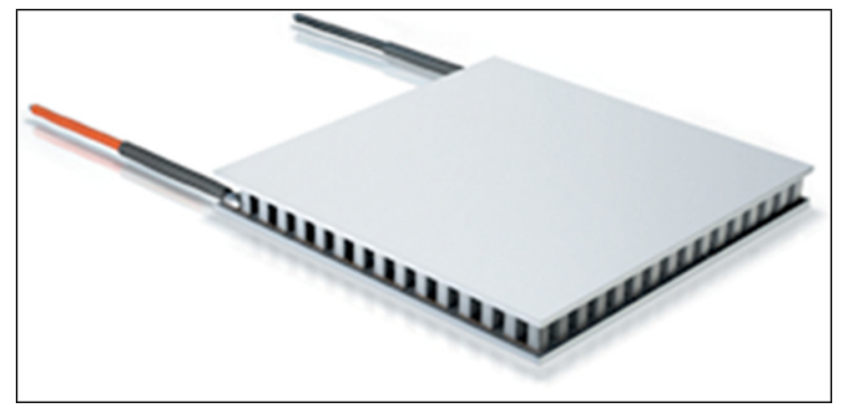

Fig. 5. Single module of TEC [7].

Multistage module highly required when the temperature different is large. The multistage design essentially place two or more single module which stacked on each other just like figure 6 [10]. Increasing the number of stages increases the coefficient of performance for a given cold side temperature. 


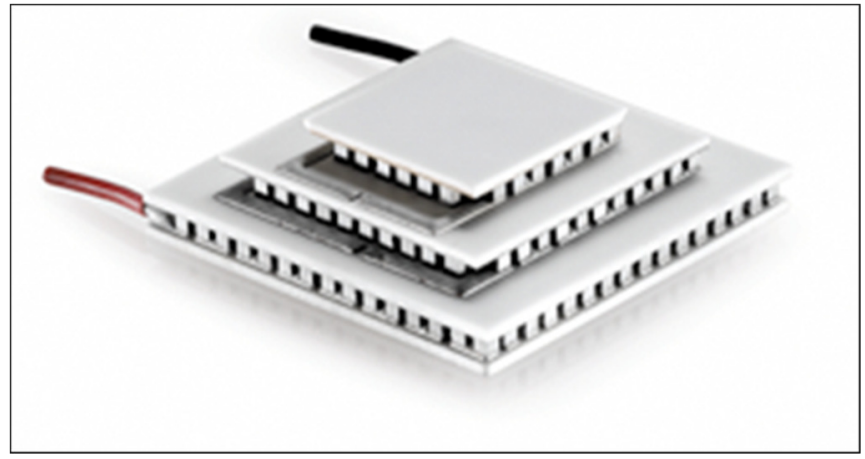

Fig. 6. Picture of multistage module of thermolelctric cooler [7].

The average (COP) of the cooling system of TEC with photovoltaic/thermal (PV/T) is calculated and the result shows that the cooling capacity of the heat pipe PV/T is about 35.77 KWh during daytime. This system is very useful in summer days because the high surrounding temperature and the heat produced by TEC will give electrical energy to the $\mathrm{PV} / \mathrm{T}[17]$.

Harman method can be used to evaluate the cooling performance of TEC by obtaining the figure of merit, ZT through direct current and transient voltage response. Nonetheless, this method does not work accurately. Therefore, a heat flow meter was developed to reduce the measurement time. By using this method, the figure of merit is expected to be achieved by $3 \%$ of deviation due to the transient cooling power, variable technique to measure and value of thermal loss [18].

In 2014, Harman method is testing for isothermal condition which is small condition and isothermal interface, flexible thermal gradient but compare to Wei Zhu et al.[18] that measure the cooling performance by the obtained the figure of merit. The configuration and its imedence of the module is including for this method because different application usage required to measure large impedance variation of the module. This method is to measure the module but it does not include the ceramic part of TEM [19].

The comparison COP between TEC and conventional cooling system is the TEC have limited usage in cooling application due to the low COP but Jaziel et al. have found that an optimal cooling with respect to the injected electric currents with $36 \%$ improvement in the performance by determining the relationship between elliptic isocontours for different temperature and thermal performance measured by thermal ZT. The isocontour obtain to apply electric current for each thermoelectric and the current flow in stage of cooler condition [20]. While the COP of conventional cooling system is higher due to the currently technology but it is still does not reduce the electricity usage if the system is running continuously.

\subsection{Thermoelectric Applications}

TE device is considered as successfully commercialized due to high reliability, low weight and less maintenance. [21]. Thermoelectric offers two primary applications, one is for renewable energy utilization and second is for generating electricity with waste energy during heating or cooling process. This module can operate as a cooler or electricity generator. Cooler mode allows the module to transform the current flow to the the temperature difference.direct current to temperature gradient. Meanwhile, electricity generator mode allows the module to produce electricity caused by the temperature gradient. 


\subsubsection{Air Conditioner}

Thermoelectric air conditioner is simple, convenient system and environmental friendly [1]. The cooling and heating mode is easily changed by reversing the current input. Nevertheless, the system is costly. The low (COP) of thermoelectric is the factor that limits its application for domestic cooling even though the thermoelectric air conditioner (TEAC) is noiseless and compact. The COP of cooling performance of an air conditioner can highly performance depends on the absorption of heat from thermoelectric converter.

\subsubsection{Refrigerator}

A refrigerator installed with a TE system used Peltier cooling principle to provide a quick cooling cycle which can reduces theof electrical charges in the thermoelectric material. This principle is noiseless, reliable, has low cost of production and has the merit of being light. One interesting additional application for refrigerator where the solar cell operated in tandem with thermoelectric device through photovoltaic effect that receive solar energy from solar cell and then converts it to electricity [1].

\subsubsection{Electronic Cooling Device}

An ice-free portable cooling system such as a picnic cooler and portable food and beverages cooler can be implemented with a TEC module. The thermoelectric system is also used to control the cooling of an electronic components of industrial equipments [22] with limited cooling capacity that less than 1000W [23].

\subsubsection{Power Generation}

Thermoelectric modules can also be used as a power generator. Recent researcher evaluated the performance of TE module in terms of consistency, power output and conversion efficiency [24]. The cost can be reduced to $0.02 / \mathrm{kWh}$. Thermoelectric generator is to produce electricity by harvesting waste heat from the thermoelectric system [1].

Therefore, the heat waste also can generate high potential electric. Due to the electricity produced the steelwork industry and automobile engines are applied the heat waste as as energy sources. Sharma et al. [5] has reviewed that the electrical power generation of thermoelectric is able to generate thermoelectric refrigerator and heat pump.

\subsubsection{Hybrid System}

Sharma et al. [5] had experimented the hybrid system with vapor compression and thermoelectric system. The performance of the thermoelectric air conditioner has a large potential market because of its simplicity, reliabilty and environmental friendly. However, the cost of this system is expensive.

Although the COP of thermoelectric air conditioner is low, it can used for small enclosures such as car and submarine. Some researchers analyzed the performance of the thermoelectric cooling attached with a solar cell and they found that the waste heat was able to produce temperature gradient which is appropriate for application that control temperature $[5,25]$. 


\subsection{Commersial TEC Air Conditioner (TEAC)}

In our daily life, the refrigeration is required for many applications and Peltier Effect is more interesting for air conditioner. The efficiency of an air conditioner is measured by the COP, the rate of heat transfer and the maximum value of temperature that can be reached from hot side and the cold side. The COP depends on the figure of merit of semiconductor material [26].

TEAC is widely used in the lower cooling capacity because it can be powered directly with a DC supply. The TEAC is also convenient, light, easy to control and can be set up quickly. S.B.Riffat and Guoquan Qie reported that the range of operating temperature is about $-40 \square$ to $70^{\circ} \mathrm{C}$ and can directly powered to sources or photovoltaic cell [27].

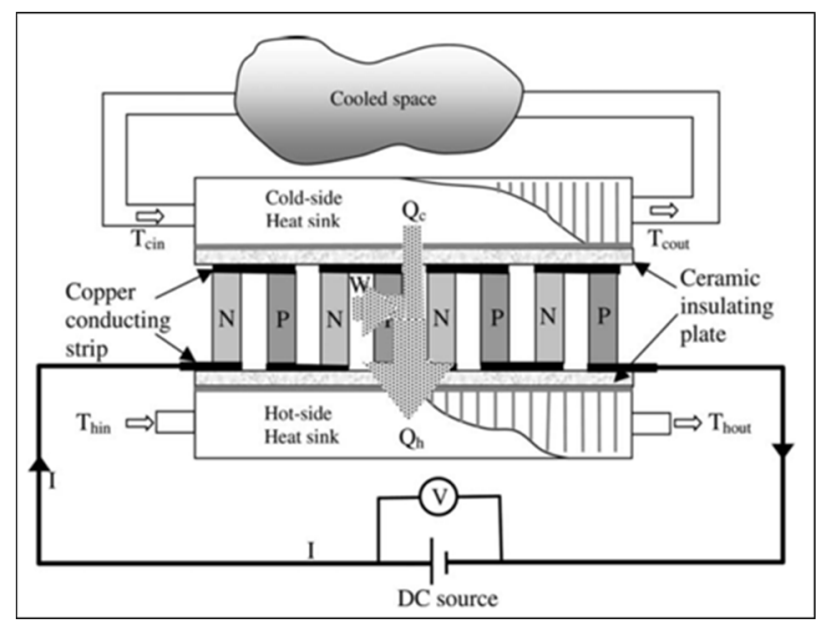

Fig. 7. Schematic of TEAC [27].

Figure 7 shows the process of removing heat from the room which is then utilized by the air conditioner which produces a cooling effect to the refrigerant. However, the refrigerant used in the air conditioner may cause the release of harmful gases such as Freon, Ammonia and chlorofluorocarbons. By installing the TE module to the air conditioner might reduce the air pollution as it is an eco-friendly device [28].

Benziger and Anu Nair [28] conclude that the lower power input can be obtained at higher COP so the rate of energy consumption can be reduced. Tipsaenporm et al. [29] reported that the thermoelectric cooling capacity will proportionally increase depending on the current electrical input and ratio of thermoelectric element.

\subsection{Discussion}

TEC is the module that can be applied in different areas due to its functionality that gives many advantages. There are no limitations of TEC usage in any application. However, the improvement of the $\mathrm{COP}$ of the cooling system has been made and the researcher found that the COP is increased when the cooling capacity is increase [29].

The TEC module has been implemented in medical and scientific application, automotive heating and cooling, and air conditioning integrated with PV solar. The application of thermoelectric has been summarized in certain characteristic such as an electronic device, automobile application, local refrigeration and scientific usage [30]. However, the application of TEC is not limited because the material is made of high performance efficiency. 
The comparison of COP between TEC and conventional air conditioner has been made which resulting the range of actual COP is 2.6-3.0 and 0.38-0.45 respectively [30]. However, optimization on the TEAC needed to be done to increase the cooling performance in a higher cooling range by reducing usage of cooling capacity. The corresponding experimental data will be used for validation purpose. The experimental validation offers the expected accuracy level quantification and demonstrates the reliability of the model in the practical conditions.

\section{Conclusion}

An extensive literature review has been carried out on the cooling performance of TEC and its application to appliances. At this point, the specific result of the flexibility aspect of TEAC technique found that the solution about the conventional air conditioner problem such as noise, size and vibration can be overcome by TE module. For many years, Bismuth Telluride (BiTe) is the best ZT that equal to 1 which the efficiency is about 5-7\% for most application but still not suitable for temperature over $230 \square$ because it can fall off rapidly at the hot side of TEC [11]. However, the performance of BiTe is still classified low so the another material should be studied to get better performance of TEC. In addition, TEC has not affected the environment since there is zero emission of carbon dioxide gas. The TEC is widely used as a cooling source for many applications. TEC would be an effective cooling and heating device with the proper design system. Finally, the proper mathematical model of TEC need to obtain for more effectiveness performance of cooling and heating specially to determining the thermal performance under actual working environment. The appropriate control design required to be implemented.

The authors would like to acknowledge the Ministry of Higher Education of Malaysia and the Universiti Malaysia Perlis, Perlis, Malaysia, for the financial support under FRGS (9003-00564)

\section{References}

1. M.H. Elsheikh, D. Shnawah, M. Sabri, S. Said, M. H. Hassan, M.A. Bashir and M. Mohamad, Renew. Sust. Energ. Rev., 30, 337-355, (2014)

2. X. Zheng, C. Liu, Y. Yan, Q. Wang, Renew. Sust. Energ. Rev., 32, 486-503, (2014)

3. E. Nogueira, J.R. Camargo, Rev.Cienc. Exatas. Taubate, 9/10, (2004)

4. Thermoelectric Module. Available: https://thermal.ferrotec.com/technology/

5. S. Sharma, V.K. Dwivedi, S.N. Pandit, Int J. of Green Energy, 11, 899-909, (2014)

6. M.H. Elsheikh, D.A. Shnawah, M.F.M. Sabri, S.M. Said, M.H. Hasssan, M.B.A Bashir, M. Mohamad, Renew. Sust. Energ. Rev., 30, 337-355, (2014)

7. Thermoelectric Modules Deep-Cooling Multi-stage Peltier Coolers. Available: https://thermal.ferrotec.com/products/peltier-thermoelectric-cooler-modules/deepcooling/

8. Peltier. Available: https://quantumtunnel.wordpress.com/2012/12/14/peltier-effect-sciadvent-day-13/peltier_effect/\#main.

9. Yaakov Kraftmakher, Eur. J. Phys., 26, 956-967, (2005)

10. S. Twaha, J.Zhu, Y.Yan, B.Li, Renew. Sust. Energ. Rev., 65, 698-726, (2016)

11. B. Yang, H. Ahuja, T. N. Tran, 14, (2008)

12. G. Tan, D. Zhao, Appl. Therm. Eng., 86, 187-198, (2015)

13. D. Enescu, E. O.Virjoghe, Renew. Sust. Energ. Rev., 38 903-916, (2014)

14. B. Huang, C. Chin, C. Duang, Int.J. of Refrigeration, , 23, 208-218, (2000)

15. K. Lindler, Energ. Convers. Manage., 39, 1009-1014, (1998)

16. D. Enescu, E. Virjoghe, Renew. Sust. Energ. Rev., 38, 903-916, (2014)

17. W. He, J. Zhou, C. Chen and J. Ji, Energ. Convers. Manage., 84, 41-49, (2014) 
18. W. Zhu, Y. Deng, Y. Wang and A. Wang, Microelectr. J., 44, 860-868, (2013)

19. H. Wang, R. Mccarty, J. R. Salvador, A. Yamamoto, and J. K. Nig, J. Electron. Mater., (2014)

20. J. A. Rojas, I. Rivera, A. Figueroa and F. Vázquez, Entropy, 18, 255 (2016)

21. W. He, J. Zhou, J. Hou, C. Chen and J. Ji, Appl. Energ., 107, 89-97, (2013)

22. D. Enescu, E. Virjoghe, Renew. Sust. Energ. Rev., 38, 903-916, (2014)

23. L. Bell, Science, 321, 1457-1461, (2008)

24. D. Rowe, G. Min, J. Power Sources, 73, 193-198, (1998)

25. D. Zhao, G. Tan, Appl. Therm. Eng., 66, 15-24, (2014)

26. E. Nogueira, J. Rui Camargo, 910, 9-11, (2016)

27. S. Riffat, G. Qiu, Appl. Therm. Eng., 24, 1979-1993, (2004)

28. B. B, N International Conference on Engineering and Scientific Innovations(2015)

29. W. Tipsaenporm, C. Lertsatitthanakorn, B. Bubphachot, M. Rungsiyopas, S. Soponronnarit, J. Electron. Mater., 41, 186-1192, (2012)

30. D. Zhao, G. Tan, Appl. Therm. Eng., vol. 66, 15-24, (2014) 